\title{
Unraveling the electric field-induced second harmonic generation responses of stilbazolium ion pairs complexes in solution using a multiscale simulation method
}

Tárcius N. Ramos ${ }^{1,2}$, Sylvio Canuto ${ }^{1}$, Benoît Champagne ${ }^{2 *}$

${ }^{1}$ Universidade de Sao Paulo, Instituto de Fisica, Rua do Matão 1371, 05508-090, São Paulo, SP, Brazil.

2 Theoretical Chemistry Lab, Unit of Theoretical and Structural Physical Chemistry, Namur Institute of Structured Matter, University of Namur, rue de Bruxelles, 61, B-5000 Namur, Belgium.

* Email: benoit.champagne@unamur.be 
Table S1. Basis set effects on the $\left[\mu \beta_{/ /}\right]_{e f f}\left(10^{-48} \mathrm{esu}\right)$ response at $1907 \mathrm{~nm}$ as determined at the IEFPCM/TD-DFT/M06-2X for complexes 1-4 and different counterion positions.

\begin{tabular}{|l|c|c|c|c|c|c|c|c|}
\hline Basis set & \multicolumn{2}{|c|}{1} & \multicolumn{2}{c|}{ 2 } & \multicolumn{2}{c|}{3} & \multicolumn{2}{c|}{4} \\
\hline & In plane & Out of plane & In plane & Out of plane & In plane & Out of plane & In plane & Out of plane \\
\hline 6-311+G(d) & 227.3 & 58.6 & 520.3 & 151.8 & 2206.3 & 1061.1 & 2285.1 & 1301.5 \\
\hline 6-311+G(d,p) & 225.4 & 58.0 & 522.0 & 153.6 & 2227.1 & 1074.6 & 2308.1 & 1317.4 \\
\hline aug-cc-pVDZ & 219.1 & 60.4 & 516.7 & 155.6 & 2256.3 & 1096.4 & 2345.8 & 1352.3 \\
\hline
\end{tabular}

Table S2. Root-mean-square deviations (RMSD) between the atomic positions of the minimum energy geometries evaluated at the $\omega$ B97X-D level and those obtained at the MP2 and reparameterized OPLS-AA (in parenthesis) levels. BLA and the $\mathrm{d}_{\mathrm{NA}}$ values obtained from MP2, $\omega \mathrm{B} 97 \mathrm{X}-\mathrm{D}$ and OPLS-AA optimized geometries. All values are given in $\AA$. The molecules were aligned using the quaternion algorithm.

\begin{tabular}{|c|c|c|c|c|c|c|c|}
\hline \multirow{2}{*}{ Complex } & Atomic positions & \multicolumn{3}{|c|}{ BLA } & \multicolumn{3}{c|}{$\mathrm{d}_{\mathrm{NA}}$} \\
\hline & RMSD & MP2 & $\omega$ B97X-D & OPLS-AA & MP2 & 0 97X-D & OPLS-AA \\
\hline $\mathbf{1}$ & $0.04(0.14)$ & 0.111 & 0.117 & 0.117 & 4.35 & 4.50 & 4.49 \\
\hline $\mathbf{2}$ & $0.05(0.13)$ & 0.099 & 0.115 & 0.115 & 4.35 & 4.50 & 4.58 \\
\hline $\mathbf{3}$ & $0.19(0.16)$ & 0.090 & 0.098 & 0.096 & 4.35 & 4.51 & 4.58 \\
\hline $\mathbf{4}$ & $(0.23)$ & - & 0.095 & 0.096 & - & 4.51 & 4.49 \\
\hline $\mathbf{4 . 1}$ & $(0.19)$ & - & 0.095 & 0.096 & - & 3.37 & 3.45 \\
\hline $\mathbf{4 . 2}$ & $(0.20)$ & - & 0.098 & 0.101 & - & 3.49 & 3.50 \\
\hline $\mathbf{4 . 3}$ & $(0.22)$ & - & 0.098 & 0.099 & - & 3.49 & 3.58 \\
\hline $\mathbf{4 . 4}$ & $(0.21)$ & - & 0.101 & 0.102 & - & 3.53 & 3.46 \\
\hline
\end{tabular}


Table S3. IEFPCM/TD-DFT/M06-2X/6-311+G(d) EFISHG and HRS first hyperpolarizabilities at $1907 \mathrm{~nm}$ as a function of method to optimize the geometry and of the position of the anion. The reported quantities are $\mu \beta_{/ /}\left(10^{3}\right.$ a.u.), $\beta_{/ /}$(a.u.), $\mu$ (a.u.), the $\theta_{(\mu, \beta)}$ is angle between the $\mu$ and $\beta$ vectors (deg), $\beta_{H R S}$ (a.u.) and its depolarization ratio (DR).

\begin{tabular}{|c|c|c|c|c|c|c|c|c|}
\hline Complex & $\begin{array}{c}\text { Geometry } \\
\text { optimization }\end{array}$ & Anion position & $\mu \beta_{/ /}$ & $\beta_{/ /}$ & $\mu$ & $\theta_{(\mu, \beta)}$ & $\beta_{H R S}$ & $\mathrm{DR}$ \\
\hline \multirow{4}{*}{1} & MP2 & In plane & 25.00 & 2954 & 8.46 & 58.8 & 4080 & 4.48 \\
\hline & MP2 & Out of plane & 4.71 & 769 & 6.13 & 81.1 & 3629 & 4.26 \\
\hline & $\omega B 97 X-D$ & In plane & 23.48 & 2659 & 8.83 & 60.1 & 3822 & 4.46 \\
\hline & $\omega B 97 X-D$ & Out of plane & 4.93 & 750 & 6.57 & 81.0 & 3485 & 4.31 \\
\hline \multirow{4}{*}{2} & MP2 & In plane & 57.54 & 6270 & 9.18 & 44.6 & 6232 & 4.64 \\
\hline & MP2 & Out of plane & 13.42 & 2151 & 6.24 & 74.1 & 5615 & 4.51 \\
\hline & $\omega B 97 X-D$ & In plane & 54.72 & 5744 & 9.53 & 45.5 & 5796 & 4.63 \\
\hline & $\omega B 97 X-D$ & Out of plane & 14.45 & 2141 & 6.75 & 73.4 & 5354 & 4.51 \\
\hline \multirow{4}{*}{3} & MP2 & In plane & 196.69 & 19071 & 10.31 & 37.1 & 16746 & 4.78 \\
\hline & MP2 & Out of plane & 82.76 & 11392 & 7.27 & 58.0 & 15108 & 4.72 \\
\hline & $\omega B 97 X-D$ & In plane & 235.21 & 21326 & 11.03 & 36.5 & 18586 & 4.78 \\
\hline & $\omega B 97 X-D$ & Out of plane & 110.30 & 14194 & 7.77 & 54.9 & 17320 & 4.76 \\
\hline \multirow{2}{*}{4} & $\omega B 97 X-D$ & In plane & 242.48 & 22975 & 10.55 & 44.5 & 22477 & 4.82 \\
\hline & $\omega B 97 X-D$ & Out of plane & 135.20 & 17348 & 7.79 & 54.4 & 20867 & 4.80 \\
\hline 4.1 & $\omega B 97 X-D$ & Out of plane & 155.23 & 20424 & 7.60 & 45.4 & 20259 & 4.86 \\
\hline 4.2 & $\omega B 97 X-D$ & Out of plane & 135.47 & 18054 & 7.50 & 51.7 & 20305 & 4.86 \\
\hline 4.3 & $\omega B 97 X-D$ & Out of plane & 94.25 & 13996 & 6.73 & 60.3 & 19770 & 4.78 \\
\hline 4.4 & $\omega B 97 X-D$ & Out of plane & 114.97 & 20308 & 5.66 & 41.1 & 18804 & 4.83 \\
\hline
\end{tabular}


Table S4. Analyses of EFISHG responses at $1907 \mathrm{~nm}$ as determined at the IEFPCM/TD-DFT/M06$2 \mathrm{X} / 6-311+\mathrm{G}(\mathrm{d})$ level as a function of method to optimize the geometry and of the position of the anion. The following quantities are considered: $\mu \beta_{/ /} / k T, \gamma_{/ /}$and $\gamma_{E F I S H G}\left(10^{4}\right.$ a.u.); $\left[\mu \beta_{/ /}\right]_{e f f}\left(10^{-48} \mathrm{esu}\right)$, $R_{3 / 2}=\left(3 k T \times \gamma_{/ /}\right) / \mu \beta_{/ /} . \mathrm{T}=298.15 \mathrm{~K}, 3 \mathrm{kT}=2.833 \times 10^{-3}$ a.u.

\begin{tabular}{|c|c|c|c|c|c|c|c|}
\hline Complex & $\begin{array}{c}\text { Geometry } \\
\text { optimization }\end{array}$ & Anion position & $\mu \beta_{/ /} / 3 k T$ & $\gamma_{/ /}$ & $\gamma_{E F I S H G}$ & $R_{3 / 2}$ & {$\left[\mu \beta_{/ /}\right]_{e f f}$} \\
\hline \multirow{4}{*}{1} & MP2 & In plane & 883 & 52.1 & 935 & 0.06 & 242.2 \\
\hline & MP2 & Out of plane & 166 & 56.7 & 223 & 0.34 & 57.8 \\
\hline & $\omega B 97 X-D$ & In plane & 829 & 48.3 & 877 & 0.06 & 227.3 \\
\hline & $\omega B 97 X-D$ & Out of plane & 174 & 52.0 & 226 & 0.30 & 58.6 \\
\hline \multirow{4}{*}{2} & MP2 & In plane & 2031 & 82.2 & 2114 & 0.04 & 547.8 \\
\hline & MP2 & Out of plane & 474 & 82.0 & 556 & 0.17 & 144 \\
\hline & $\omega B 97 X-D$ & In plane & 1931 & 75.6 & 2007 & 0.04 & 520.3 \\
\hline & $\omega B 97 X-D$ & Out of plane & 510 & 75.7 & 586 & 0.15 & 151.8 \\
\hline \multirow{4}{*}{3} & MP2 & In plane & 6944 & 200.1 & 7144 & 0.03 & 1851.5 \\
\hline & MP2 & Out of plane & 2922 & 189.6 & 3111 & 0.06 & 806.4 \\
\hline & $\omega B 97 X-D$ & In plane & 8304 & 208.8 & 8513 & 0.03 & 2206.3 \\
\hline & $\omega B 97 X-D$ & Out of plane & 3894 & 200.5 & 4094 & 0.05 & 1061.1 \\
\hline \multirow{2}{*}{4} & $\omega B 97 X-D$ & In plane & 8560 & 256.7 & 8817 & 0.03 & 2285.1 \\
\hline & $\omega B 97 X-D$ & Out of plane & 4773 & 248.7 & 5022 & 0.05 & 1301.5 \\
\hline 4.1 & $\omega B 97 X-D$ & Out of plane & 5480 & 227.5 & 5708 & 0.04 & 1420.3 \\
\hline 4.2 & $\omega B 97 X-D$ & Out of plane & 4783 & 230.1 & 5013 & 0.05 & 1239.5 \\
\hline 4.3 & $\omega B 97 X-D$ & Out of plane & 3327 & 228.4 & 3556 & 0.07 & 862.3 \\
\hline 4.4 & $\omega B 97 X-D$ & Out of plane & 4059 & 221.5 & 4280 & 0.05 & 1051.9 \\
\hline
\end{tabular}


Table S5. Charge analysis for complex 1 as a function of the method for geometry optimization (MP2 versus $\omega \mathrm{B} 97 \mathrm{X}-\mathrm{D})$ where the anion lies in the same plane as the cation ring. All results were obtained employing IEFPCM/M06-2X/aug-cc-pVDZ/CHELPG and are given in $e$.

\begin{tabular}{|c|c|c|c|c|}
\hline & Atom & MP2 & $\omega B 97 X-D$ & $\Delta(\mathrm{MP} 2-\omega \mathrm{B} 97 \mathrm{X}-\mathrm{D})$ \\
\hline 1 & $\mathrm{C}$ & 0.14 & 0.11 & 0.03 \\
\hline 2 & C & -0.33 & -0.28 & -0.06 \\
\hline 3 & C & 0.42 & 0.36 & 0.06 \\
\hline 4 & C & -0.32 & -0.29 & -0.03 \\
\hline 5 & C & 0.10 & 0.08 & 0.02 \\
\hline 6 & $\mathrm{H}$ & 0.12 & 0.13 & 0.00 \\
\hline 7 & $\mathrm{H}$ & 0.20 & 0.18 & 0.02 \\
\hline 8 & $\mathrm{H}$ & 0.18 & 0.18 & 0.00 \\
\hline 9 & $\mathrm{H}$ & 0.15 & 0.15 & 0.00 \\
\hline 10 & C & -0.31 & -0.25 & -0.06 \\
\hline 11 & C & -0.13 & -0.17 & 0.03 \\
\hline 12 & C & 0.20 & 0.25 & -0.05 \\
\hline 13 & C & -0.19 & -0.23 & 0.03 \\
\hline 14 & C & -0.21 & -0.24 & 0.04 \\
\hline 15 & C & -0.06 & -0.03 & -0.03 \\
\hline 16 & $\mathrm{H}$ & 0.13 & 0.13 & -0.01 \\
\hline 17 & C & -0.05 & -0.01 & -0.04 \\
\hline 18 & $\mathrm{H}$ & 0.13 & 0.14 & 0.00 \\
\hline 19 & C & -0.10 & -0.14 & 0.04 \\
\hline 20 & $\mathrm{H}$ & 0.10 & 0.10 & 0.00 \\
\hline 21 & $\mathrm{H}$ & 0.10 & 0.09 & 0.01 \\
\hline 22 & $\mathrm{H}$ & 0.10 & 0.11 & -0.01 \\
\hline 23 & $\mathrm{H}$ & 0.17 & 0.15 & 0.02 \\
\hline 24 & $\mathrm{H}$ & 0.15 & 0.15 & 0.00 \\
\hline 25 & C & -0.11 & -0.09 & -0.01 \\
\hline 26 & $\mathrm{H}$ & 0.09 & 0.09 & 0.00 \\
\hline 27 & $\mathrm{H}$ & 0.12 & 0.11 & 0.01 \\
\hline 28 & $\mathrm{H}$ & 0.10 & 0.09 & 0.00 \\
\hline 29 & $\mathrm{~N}$ & 0.02 & 0.04 & -0.02 \\
\hline 30 & 1 & -0.90 & -0.91 & 0.01 \\
\hline
\end{tabular}
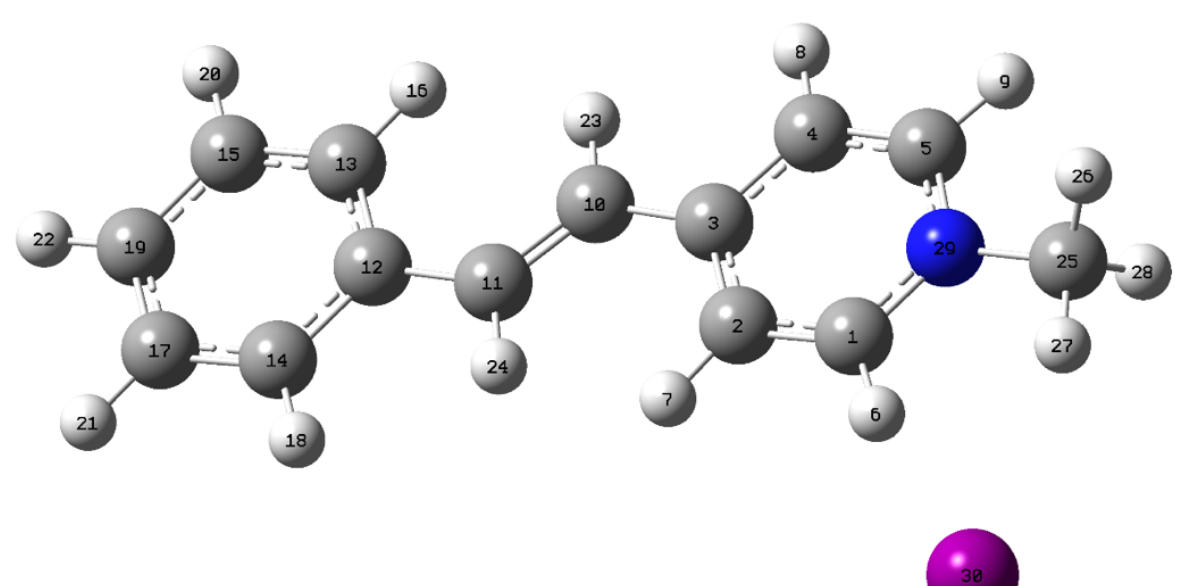
Table S6. Charge analysis for complex 2 as a function of the method for geometry optimization (MP2 versus $\omega \mathrm{B} 97 \mathrm{X}-\mathrm{D})$ where the anion lies in the same plane as the cation ring. All results were obtained employing IEFPCM/M06-2X/aug-cc-pVDZ/CHELPG and are given in $e$.

\begin{tabular}{|c|c|c|c|c|c|c|c|c|c|}
\hline & Atom & MP2 & $\omega B 97 X-D$ & $\begin{array}{c}\Delta(\mathrm{MP} 2- \\
\omega \mathrm{B} 97 \mathrm{X}-\mathrm{D})\end{array}$ & & Atom & $\mathrm{MP2}$ & $\omega \mathrm{B} 97 \mathrm{X}-\mathrm{D}$ & $\begin{array}{c}\Delta(\mathrm{MP} 2- \\
\omega \mathrm{B} 97 \mathrm{X}-\mathrm{D})\end{array}$ \\
\hline 1 & $\mathrm{C}$ & 0.07 & 0.08 & -0.01 & 22 & $\mathrm{H}$ & 0.17 & 0.17 & 0.00 \\
\hline 2 & $\mathrm{C}$ & -0.28 & -0.29 & 0.01 & 23 & $\mathrm{H}$ & 0.14 & 0.13 & 0.01 \\
\hline 3 & $\mathrm{C}$ & 0.36 & 0.37 & -0.01 & 24 & $\mathrm{C}$ & -0.11 & -0.09 & -0.02 \\
\hline 4 & $\mathrm{C}$ & -0.29 & -0.30 & 0.01 & 25 & $\mathrm{H}$ & 0.10 & 0.10 & 0.00 \\
\hline 5 & $\mathrm{C}$ & 0.14 & 0.15 & -0.01 & 26 & $\mathrm{H}$ & 0.10 & 0.09 & 0.00 \\
\hline 6 & $\mathrm{H}$ & 0.15 & 0.14 & 0.01 & 27 & $\mathrm{H}$ & 0.12 & 0.10 & 0.01 \\
\hline 7 & $\mathrm{H}$ & 0.18 & 0.18 & 0.00 & 28 & $\mathrm{~N}$ & 0.02 & 0.02 & 0.00 \\
\hline 8 & $\mathrm{H}$ & 0.18 & 0.18 & 0.00 & 29 & $\mathrm{I}$ & -0.89 & -0.91 & 0.01 \\
\hline 9 & $\mathrm{H}$ & 0.12 & 0.12 & 0.01 & 30 & $\mathrm{C}$ & 0.37 & 0.36 & 0.01 \\
\hline 10 & $\mathrm{C}$ & -0.28 & -0.29 & 0.01 & 31 & $\mathrm{C}$ & -0.26 & -0.23 & -0.03 \\
\hline 11 & $\mathrm{C}$ & -0.14 & -0.12 & -0.02 & 32 & $\mathrm{H}$ & 0.04 & 0.04 & 0.01 \\
\hline 12 & $\mathrm{C}$ & 0.18 & 0.19 & -0.01 & 33 & $\mathrm{H}$ & 0.07 & 0.07 & 0.01 \\
\hline 13 & $\mathrm{C}$ & -0.16 & -0.17 & 0.01 & 34 & $\mathrm{H}$ & 0.03 & 0.02 & 0.01 \\
\hline 14 & $\mathrm{C}$ & -0.20 & -0.20 & 0.00 & 35 & $\mathrm{C}$ & -0.22 & -0.28 & 0.06 \\
\hline 15 & $\mathrm{C}$ & -0.18 & -0.18 & 0.00 & 36 & $\mathrm{H}$ & 0.05 & 0.07 & -0.02 \\
\hline 16 & $\mathrm{H}$ & 0.12 & 0.12 & 0.00 & 37 & $\mathrm{H}$ & 0.04 & 0.06 & -0.02 \\
\hline 17 & $\mathrm{C}$ & -0.12 & -0.15 & 0.02 & 38 & $\mathrm{H}$ & 0.04 & 0.06 & -0.02 \\
\hline 18 & $\mathrm{H}$ & 0.13 & 0.13 & 0.00 & 39 & $\mathrm{C}$ & -0.22 & -0.22 & 0.00 \\
\hline 19 & $\mathrm{C}$ & 0.03 & 0.07 & -0.03 & 40 & $\mathrm{H}$ & 0.02 & 0.02 & 0.00 \\
\hline 20 & $\mathrm{H}$ & 0.15 & 0.15 & 0.00 & 41 & $\mathrm{H}$ & 0.06 & 0.06 & 0.00 \\
\hline 21 & $\mathrm{H}$ & 0.12 & 0.12 & 0.00 & 42 & $\mathrm{H}$ & 0.04 & 0.04 & 0.00 \\
\hline
\end{tabular}

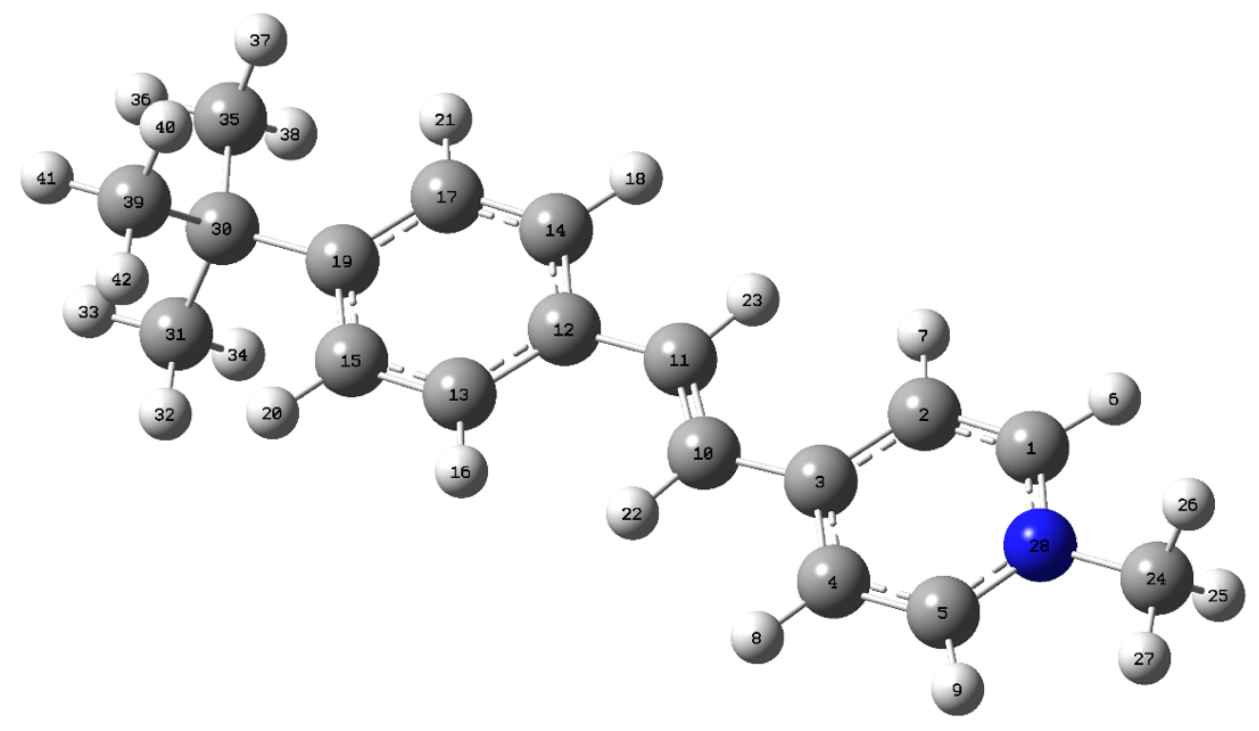


Table S7. Charge analysis for complex 3 as a function of the method for geometry optimization (MP2 versus $\omega \mathrm{B} 97 \mathrm{X}-\mathrm{D})$ where the anion lies in the same plane as the cation ring. All results were obtained employing IEFPCM/M06-2X/aug-cc-pVDZ/CHELPG and are given in $e$.

\begin{tabular}{|c|c|c|c|c|c|c|c|c|c|}
\hline & Atom & MP2 & $\omega B 97 X-D$ & $\begin{array}{c}\Delta(\mathrm{MP2}- \\
\omega \mathrm{B} 97 \mathrm{X}-\mathrm{D})\end{array}$ & & Atom & MP2 & $\omega B 97 X-D$ & $\begin{array}{r}\Delta(\mathrm{MP2}- \\
\omega \mathrm{B} 97 \mathrm{X}-\mathrm{D})\end{array}$ \\
\hline 1 & C & 0.06 & 0.06 & 0.00 & 20 & $\mathrm{H}$ & 0.16 & 0.15 & 0.01 \\
\hline 2 & C & -0.29 & -0.30 & 0.01 & 21 & $\mathrm{H}$ & 0.16 & 0.15 & 0.01 \\
\hline 3 & $C$ & 0.38 & 0.39 & -0.01 & 22 & $\mathrm{H}$ & 0.17 & 0.16 & 0.00 \\
\hline 4 & C & -0.30 & -0.31 & 0.01 & 23 & $\mathrm{H}$ & 0.13 & 0.13 & 0.00 \\
\hline 5 & C & 0.12 & 0.13 & -0.01 & 24 & C & -0.08 & -0.10 & 0.02 \\
\hline 6 & $\mathrm{H}$ & 0.15 & 0.15 & 0.00 & 25 & $\mathrm{H}$ & 0.09 & 0.09 & 0.00 \\
\hline 7 & $\mathrm{H}$ & 0.18 & 0.18 & 0.00 & 26 & $\mathrm{H}$ & 0.09 & 0.09 & -0.01 \\
\hline 8 & $\mathrm{H}$ & 0.17 & 0.18 & 0.00 & 27 & $\mathrm{H}$ & 0.11 & 0.11 & 0.00 \\
\hline 9 & $\mathrm{H}$ & 0.13 & 0.12 & 0.01 & 28 & $\mathrm{~N}$ & 0.02 & 0.03 & -0.02 \\
\hline 10 & C & -0.32 & -0.32 & 0.00 & 29 & 1 & -0.90 & -0.91 & 0.01 \\
\hline 11 & C & -0.12 & -0.12 & 0.00 & 30 & $\mathrm{~N}$ & -0.41 & -0.25 & -0.17 \\
\hline 12 & $C$ & 0.14 & 0.14 & 0.00 & 31 & $C$ & 0.15 & 0.05 & 0.10 \\
\hline 13 & C & -0.15 & -0.15 & 0.00 & 32 & $\mathrm{H}$ & 0.00 & 0.02 & -0.02 \\
\hline 14 & C & -0.19 & -0.19 & 0.00 & 33 & $\mathrm{H}$ & 0.05 & 0.07 & -0.02 \\
\hline 15 & C & -0.28 & -0.25 & -0.03 & 34 & $\mathrm{H}$ & 0.00 & 0.02 & -0.02 \\
\hline 16 & $\mathrm{H}$ & 0.13 & 0.13 & 0.00 & 35 & C & 0.09 & 0.04 & 0.05 \\
\hline 17 & C & -0.25 & -0.23 & -0.02 & 36 & $\mathrm{H}$ & 0.06 & 0.07 & -0.01 \\
\hline 18 & $\mathrm{H}$ & 0.14 & 0.13 & 0.01 & 37 & $\mathrm{H}$ & 0.01 & 0.02 & 0.00 \\
\hline 19 & C & 0.39 & 0.30 & 0.09 & 38 & $\mathrm{H}$ & 0.01 & 0.02 & -0.01 \\
\hline
\end{tabular}

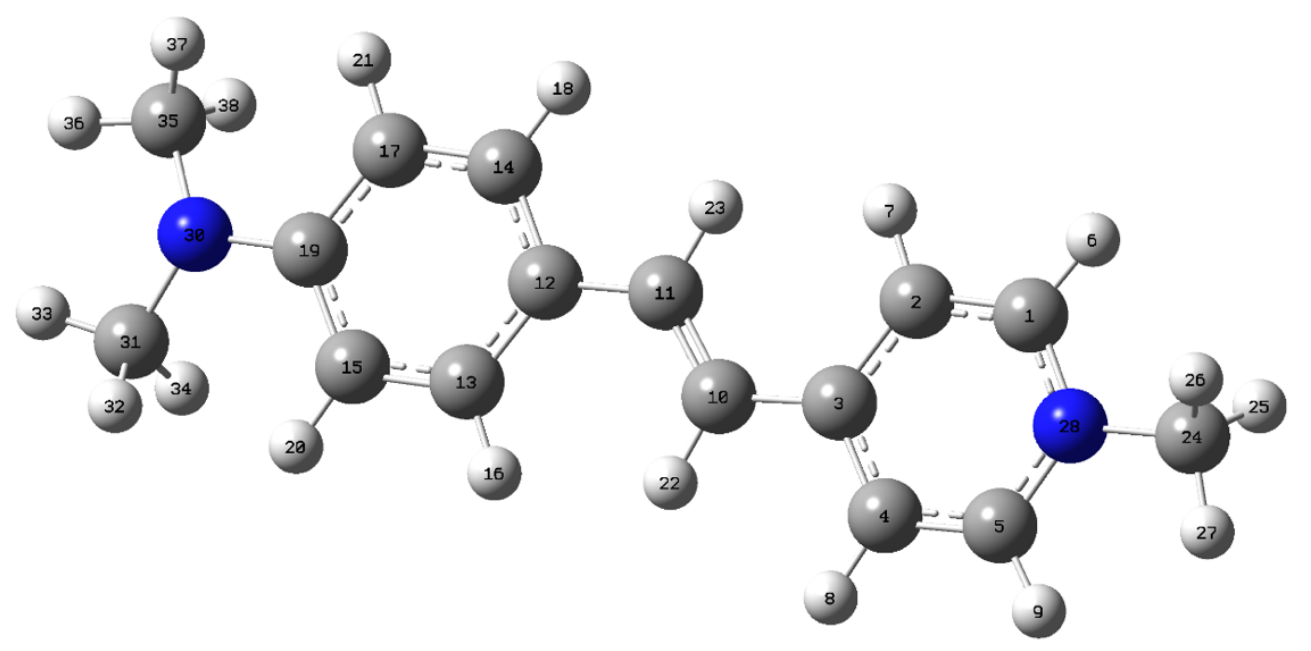



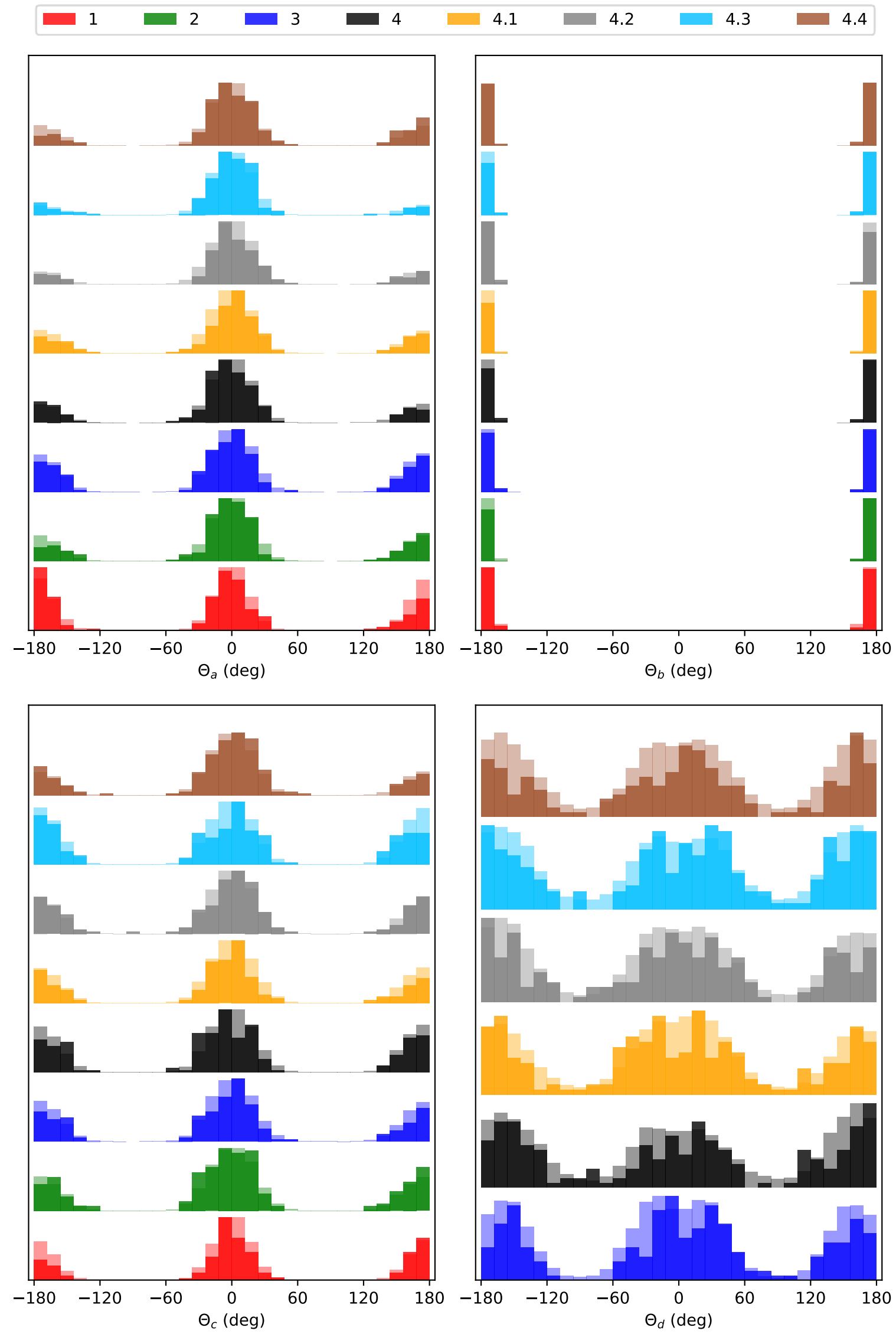

Figure S1. Distribution of the angles $\theta_{a}, \theta_{b}, \theta_{c}$, and $\theta_{d}$ of the whole simulation (translucent) and of the 200 sampled configurations (opaque). 
Complex 1

HOMO-3

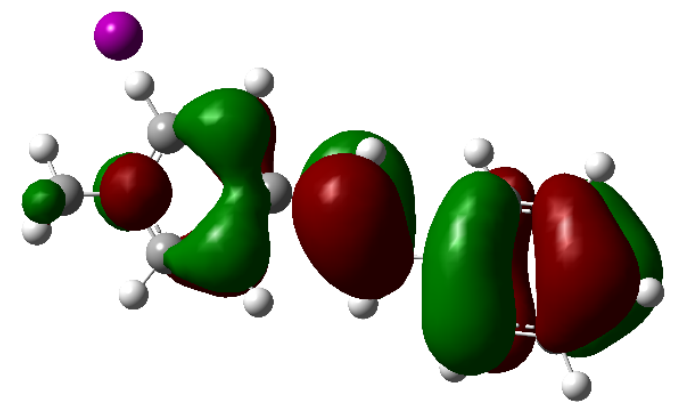

Complex 2

HOMO-3

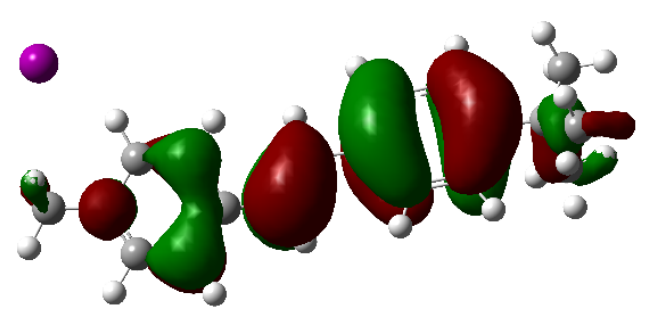

Complex 3

HOMO

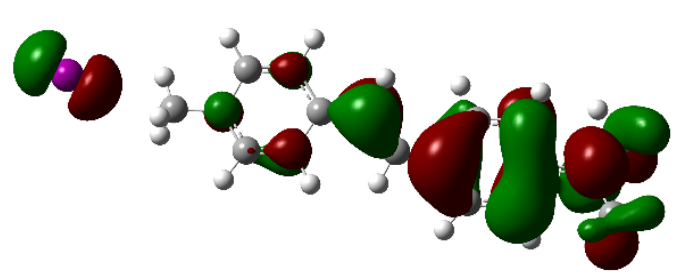

Complex 4

HOMO

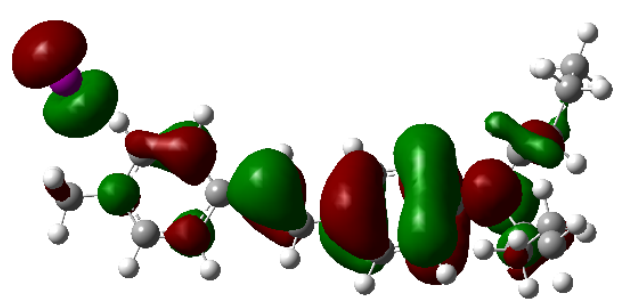

Continue.

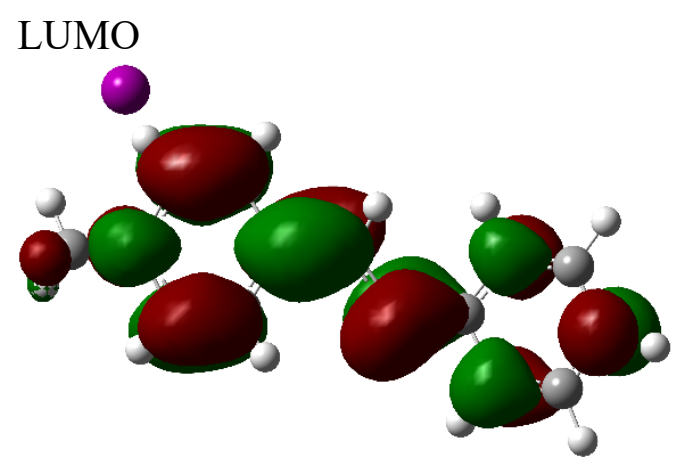

LUMO

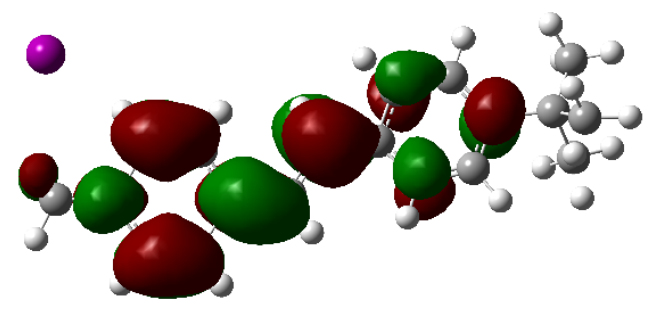

LUMO

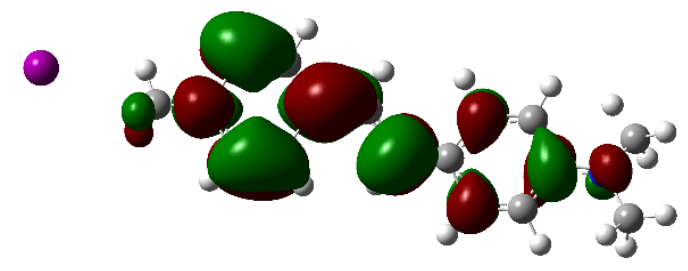

LUMO

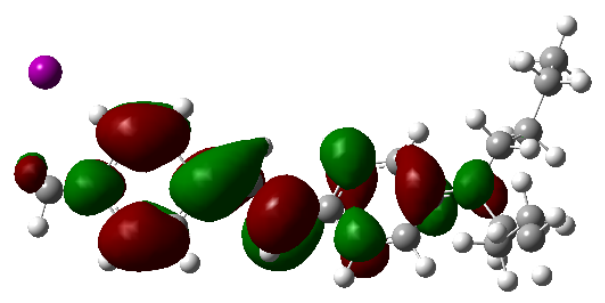




\section{Continue.}

Complex 4.1

HOMO

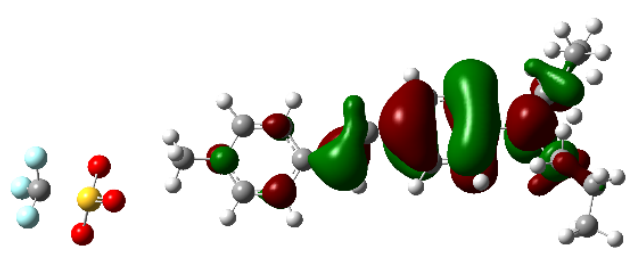

LUMO

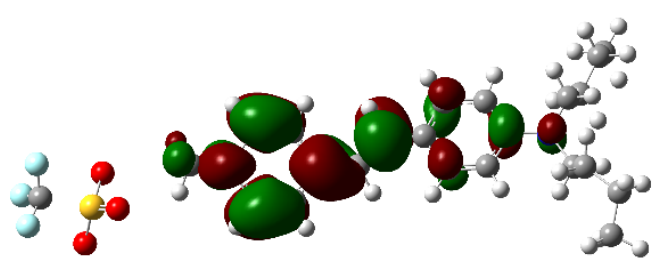

Complex 4.2

HOMO
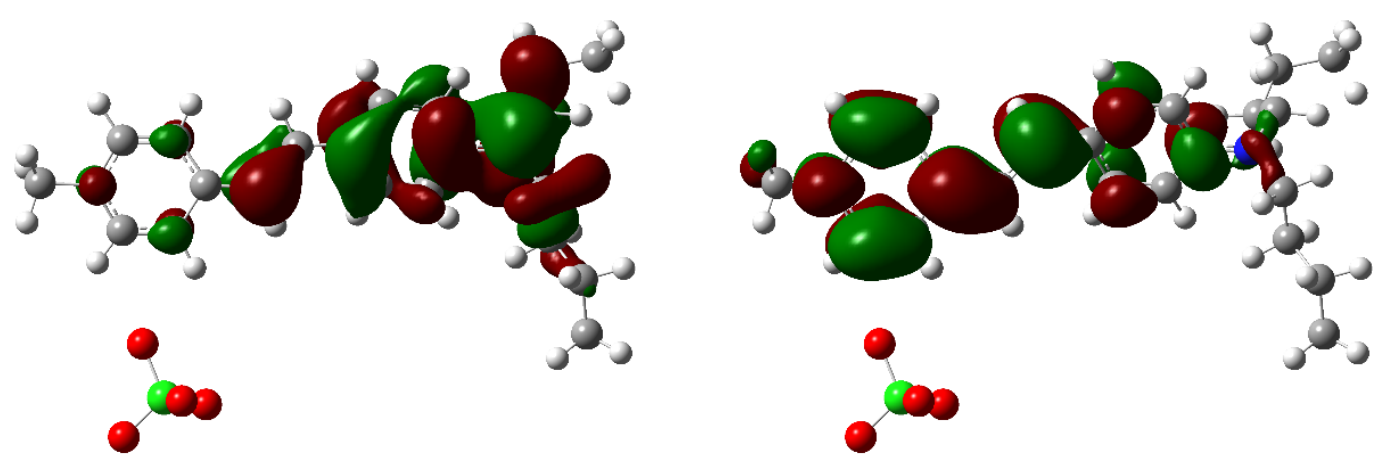

Complex 4.3

HOMO-2

LUMO
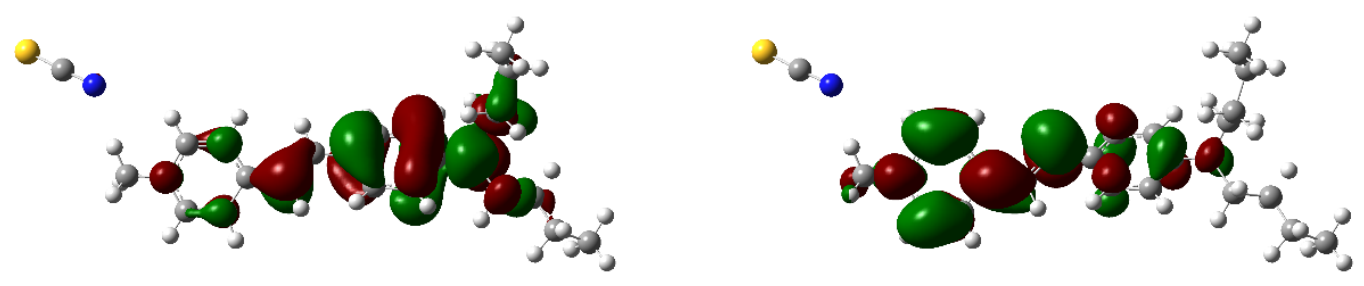

Complex 4.4

HOMO
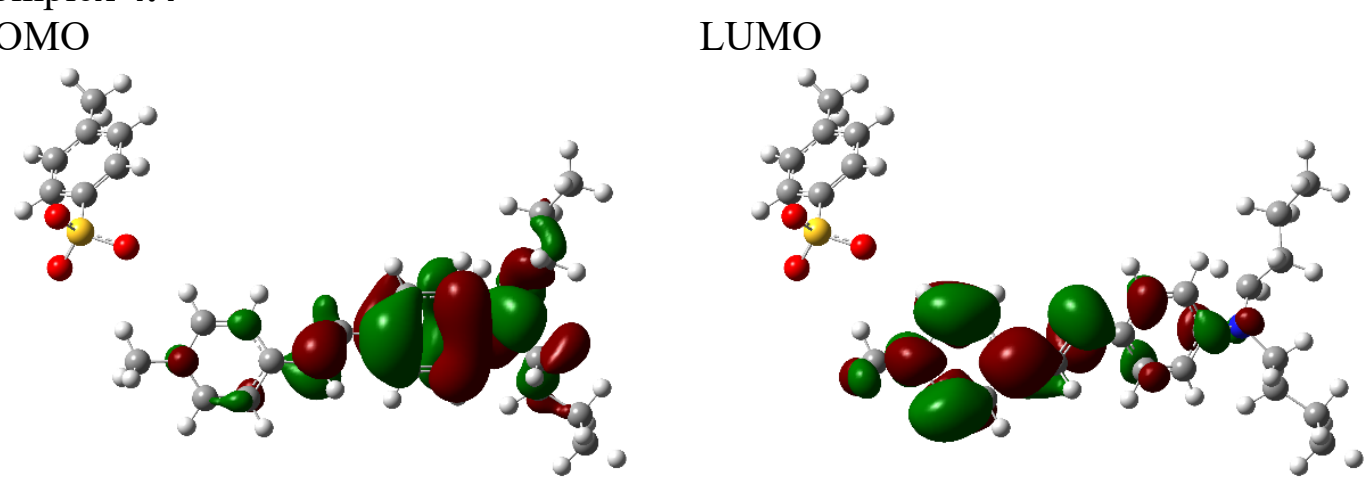

Figure S2. Main molecular orbitals involved in the dominant low-energy excitation obtained at the IEFPCM/TD-DFT/M06-2X/6-311+G(d) level. The snapshots were selected randomly from the sampled configurations. 

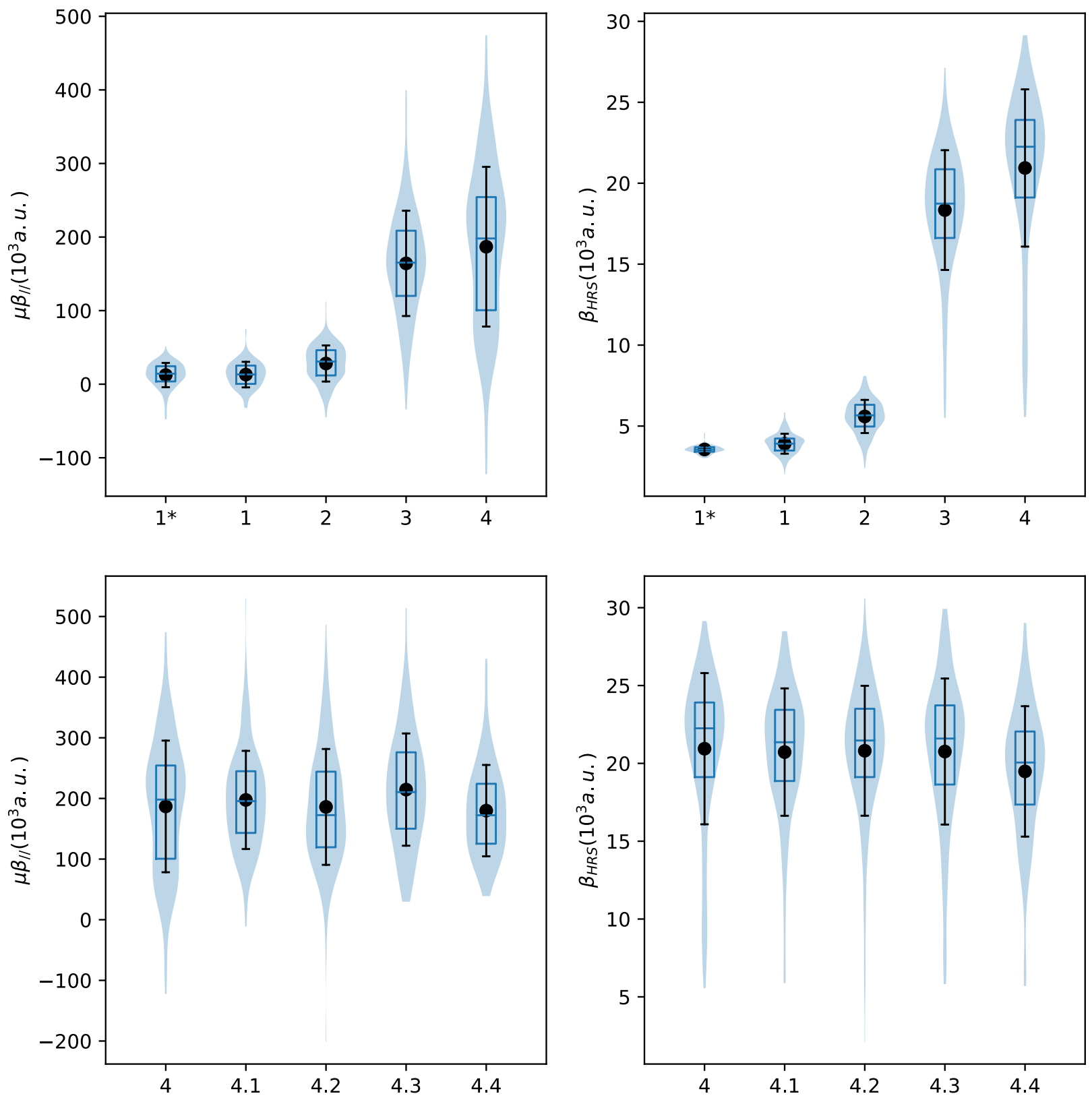

Figure S3. Violin distribution plots for $\mu \beta_{/ /}$and $\beta_{H R S}$, including the average (black point), the standard deviation (black lines) and the boxplot (blue lines indicating the first quartile at $25 \%$, the median and the third quartile at $75 \%$ ). The calculations were performed at the IEFPCM/TDDFT/M06-2X/6-311+G(d) level. 

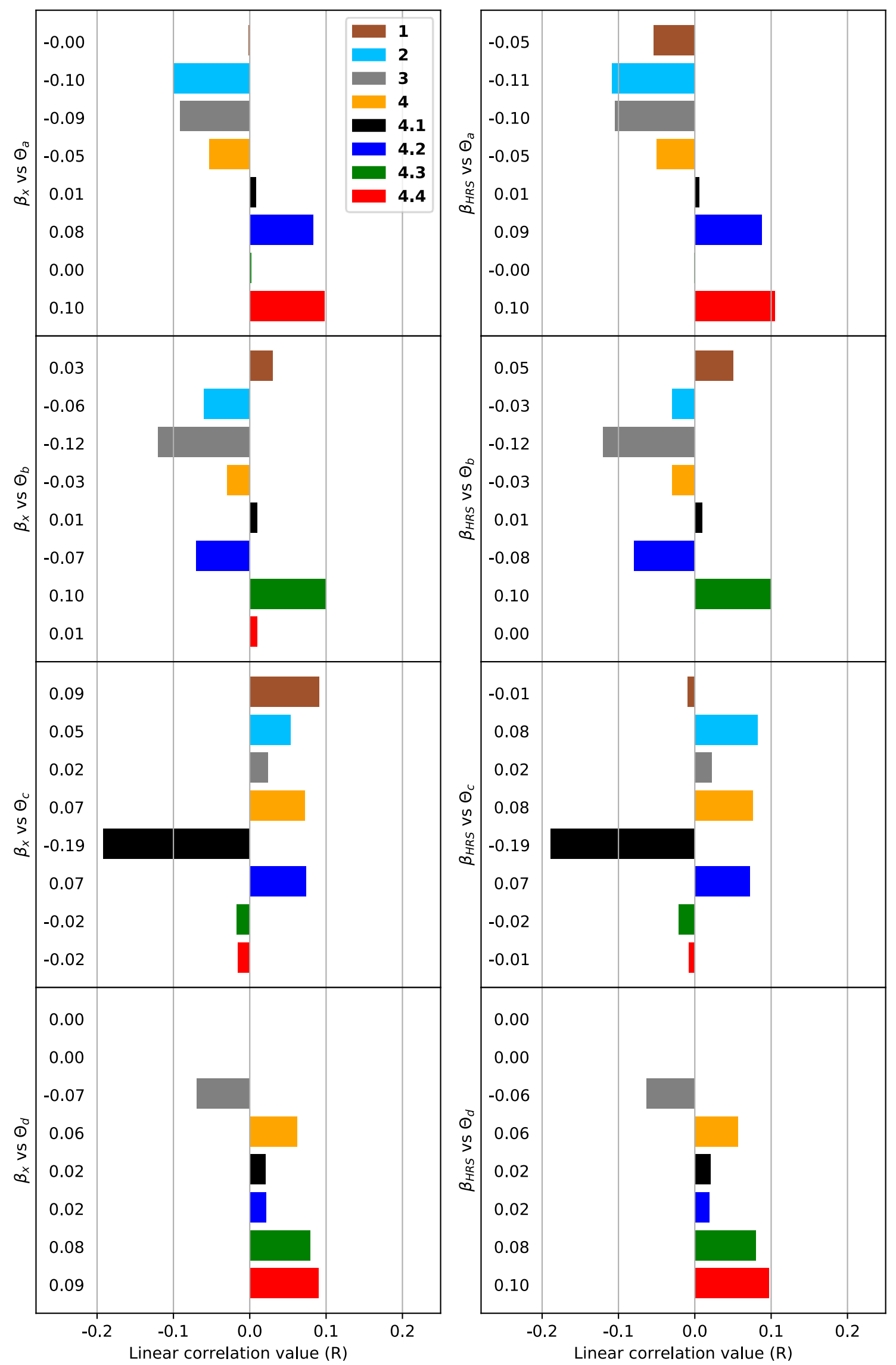

Figure S4 Linear correlation analyses for $\beta_{x}$ and $\beta_{H R S}$ versus the $\theta_{a}, \theta_{b}, \theta_{c}$, and $\theta_{d}$ torsion angles. 